\title{
Pregnant Women and Influenza H1N1
}

\author{
Kathy L. Kyser, M.D. , ${ }^{1}$ Janet I. Andrews, M.D. ${ }^{1}$
}

Key Words: Pregnancy, H1N1, Women, Influenza, Pandemic

\begin{abstract}
In 2009, uncontained transmission of novel H1N1 influenza rapidly became a worldwide pandemic and concern. Infected pregnant women were identified at high risk for H1N1 influenza complications that included an increased mortality rate that was greater than the general population affected by the disease. Major worldwide effort has been done over the last year to develop a strategy for containment of H1N1 transmission, disease prevention, and vaccination development.
\end{abstract}

1 Division of Maternal-Fetal Medicine, Department of Obstetrics and Gynecology, Carver College of Medicine, University of lowa Hospitals and Clinics.

\section{Background}

During the spring of 2009, headlines across the country spread the news that an outbreak of influenza-like illness was causing significant morbidity and mortality to the people of Mexico. By April 2009, two children in the United States were diagnosed with a novel influenza $A(H 1 N 1)$ and this novel virus was found to be the cause of the respiratory outbreaks in Mexico. This new influenza virus spread quickly throughout the world. On June 11, 2009, the World Health Organization (WHO) announced a worldwide pandemic of uncontained communitylevel transmission of novel influenza H1N1 in numerous regions of the world.

For most healthy people infected with influenza H1N1, the disease will be selflimited. Several subpopulations including pregnant women have been identified at high risk for influenza H1N1 complications. The designation of pregnancy as a high risk condition was prompted by a very recent report by the Centers for Disease Control and Prevention (CDC) published in The Lancet 2009.

The Lancet report included all cases of infection with $\mathrm{H} 1 \mathrm{~N} 1$ in pregnant women identified through the CCD between April 15 and May 18, 2009 as well as the deaths associated with this virus during the first two months of the outbreak. During the first month of the outbreak, 34 cases of confirmed or probable cases of $\mathrm{H} 1 \mathrm{~N} 1$ in pregnant women were identified and reported to the CDC. Eleven of these cases were admitted to the hospital. The admission

Please cite this paper as: Kyser KL, Andrews Jl. Pregnant Women and Influenza H1N1. Proc Obstet Gynecol. 2010 Apr;1(1):Article 7 [7 p.]. Available from: http://ir.uiowa.edu/pog/vol1/iss1/7/.

Free full text article.

Corresponding author: Kathy L. Kyser, M.D. Department of Obstetrics and Gynecology, University of lowa, 31330 PFP, 200 Hawkins Drive, lowa City, IA 52242. Telephone (319)3564839. kathy-kyser@uiowa.edu

This is an Open Access article distributed under the terms of the Creative Commons Attribution 3.0 Unported License (http://creativecommons.org/licenses/by/3.0), which permits unrestricted use, distribution, and reproduction in any medium, provided the original work is properly cited. 
rate for pregnant women infected with H1N1 was almost four times higher than the admission rate found in the general population. The only difference in the clinical symptoms between pregnant women and the general population was a higher incidence of shortness of breath. Also during the first month of the outbreak, the reported overall use of antiviral therapy increased, but only a quarter of these women started antiviral therapy in less than 48 hours of symptom onset. By the end of the first two months of the outbreak, six pregnant women in the United States had died of influenza associated complications. Four of the six deaths occurred in women who were in their third trimester. All of these women developed pneumonia followed by respiratory distress syndrome requiring mechanical ventilation.

Although the reliance on surveillance data and laboratory testing methods to determine which cases were reported do limit this study, the results strongly suggest that pregnant women infected with H1N1 are at increased risk as compared to the general population. Similar adverse outcomes in pregnant women can be found in the historical observational reports from the prior influenza pandemics of 1918 and 1957. During both of these pandemics, there was a surplus of influenza deaths among pregnant women with increased incidence of spontaneous abortion and preterm labor. In addition, multiple case reports as well as epidemiological reports show that pregnant women are also at increased risk for pregnancy complications during seasonal influenza. Thus, prompting the recommendation that all pregnant women receive the seasonal influenza vaccine.

\section{Clinical Presentation}

Pregnant women infected with influenza H1N1 present with classic symptoms of acute respiratory illness. These symptoms include the typical cough, sore throat, rhinorrhea and fever. Other less typical presenting symptoms may be body aches, headache, fatigue, vomiting and diarrhea. Many pregnant women will have a self-limited disease course, but for some, the disease progresses rapidly and may be complicated by pneumonia, with subsequent respiratory distress syndrome requiring mechanical ventilation. In less frequent cases, these complications have resulted in death. The mortality rate among pregnant women is particularly high in those affected in the third trimester.

Testing for influenza is not always necessary for a pregnant woman with fever and respiratory symptoms suspicious for flu; especially if she has had contact with others with confirmed H1N1. Rapid influenza testing is available but is poorly sensitive. Treatment decisions should not be based on a negative rapid test. Unfortunately, novel influenza H1N1 testing remains unavailable at many locations, and when it is available it may take several days before results are available. Antiviral therapy has been shown to be most effective early in the disease course; preferably within the first 48 hours of symptom onset. Because of this, the CDC strongly recommends that antiviral therapy begin as soon as possible without waiting for test results. If necessary, the University of lowa Hygienic Lab will perform PCR testing for influenza $A$ and H1N1 confirmation. 


\section{Antiviral Treatment}

All pregnant women with clinical symptoms suspicious for H1N1 should have antiviral therapy begun as soon as possible without waiting for laboratory confirmation. Early antiviral therapy has been shown to decrease the severity of symptoms, shorten the illness, and prevent serious influenza complications. Pregnancy should not be considered a contraindication to starting antiviral therapy. Benefits of these drugs in the treatment of H1N1 clearly outweigh the risk of antiviral therapy. The neuraminindase inhibitor antiviral medication zanamivir (Relenza) and oseltamivir (Tamiflu) have both been shown to shorten the duration of influenza symptoms and the severity of the disease process. Influenza H1N1 is resistant to the adamantane antiviral medications, amantadine (Symmetrel) and rimantadine (Flumadine).

Oseltamivir is the preferred antiviral agent for treating influenza H1N1. In contrast to zanamivir, oseltamivir is an oral medication and results in systemic absorption. Zanamivir is given by inhalation therapy, thereby reducing systemic absorption. Despite these differences, if oseltamivir is unavailable locally, treatment should be begun with zanamivir rather than to delay the onset of antiviral treatment. The recommended dosage of oseltamivir is a $75 \mathrm{mg}$ capsule twice per day for five days. Dosing directions for zanamivir is two $5 \mathrm{mg}$ inhalations twice per day for 5 days.

\section{Chemoprophylaxis}

When used to prevent seasonal influenza, antiviral drugs have been found to be about $70-90 \%$ effective against susceptible viruses. Pregnant women who have been in close contact with individuals who have symptoms of influenza-like illness should receive post-exposure chemoprophylaxis. As one considers which antiviral to prescribe for chemoprophylaxis, theoretically the antiviral of choice should probably be zanamivir because of its limited systemic absorption that is targeted specifically to the respiratory system. However, because of the inhaled route of administration required for zanamivir administration, the side effect profile does include possible respiratory complications. Therefore, women at known risk for respiratory problems should be prescribed oseltamivir rather than zanamivir. The recommended duration of treatment for chemoprophylaxis for both antivirals is a total of 10 days following the last known exposure to influenza H1N1. Chemoprophylaxis dose for zanamivir is two $5 \mathrm{mg}$ inhalations once per day and the dose for oseltamivir is a $75 \mathrm{mg}$ capsule once per day. Of note, with seasonal influenza, the infectious period is considered beginning one day prior to the onset of symptoms to up to 7 days after the infected person gets sick. Therefore, if the contact with the infected person occurred more than 7 days after the onset of symptoms, chemoprophylaxis is most likely not needed.

\section{Prevention}

A vaccine for H1N1 is currently in development. It is expected to be available for delivery to the public later this fall. Until this occurs, the best methods of prevention are simple steps that reduce exposure to the virus. The 
CDC general guidelines for prevention include the follow steps: (1) Frequent hand washing, (2) Minimize contact with sick individuals, (3) Have ill persons stay home except to seek medical care, (4) Cover your mouth when you cough, (5) Avoid crowded settings in communities having outbreaks of influenza H1N1, (6) use facemasks and respirators correctly, (7) Limit sharing of toys and anything else that has been in an infants mouth. After exposure to the infant's mouth, wash these items thoroughly with soap and water.

\section{Vaccine}

Vaccination has been shown to be the most effective way to prevent influenza and its complications. It is unlikely that the current seasonal influenza vaccines will prevent disease caused by novel influenza H1N1. Studies have been done that have tested the seasonal influenza vaccine with cross-reactivity antibody to novel influenza H1N1. Among adults aged 18-64 years, only $6 \%$ to $9 \%$ had titers that corresponded to a level that is considered high enough to protect them against influenza H1N1 after receiving seasonal vaccine. In those adults aged > 60 years, $33 \%$ achieved the necessary titer to protect them against novel influenza H1N1. Specific vaccines are in the development process, and are expected to be available by mid to late October 2009. It remains unclear whether the initial supply of vaccine will be enough to meet population demand. Another factor that may affect initial supply is the number of vaccine doses needed to provide adequate protection. It has been established that in children $<9$ years, two doses of seasonal influenza vaccine are required to induce immunity because young children do not have preexisting antibodies. It follows then that an additional dose of novel influenza H1N1 may also be required in children and young adults in order to provide a sufficient level of immunity. Studies are currently ongoing to determine which age groups may require the second dose of vaccine for adequate protection. An additional dose required per person may certainly affect whether there will be enough initial supply to meet demand. Because of these factors, the CDC has identified five specific groups that are considered to be at high risk for influenza H1N1 and its complications. These groups will be targeted for early vaccination.

\section{Recommendations for Vaccine Usage}

The Advisory Committee on Immunization Practices (ACIP) recommends that early vaccination should focus on five specific high risk groups. These groups include: (1) pregnant women, (2) persons who live with or provide care for infants aged $<6$ months, (3) health care workers and emergency medical services personnel, (4) persons aged 6 months - 24 years, (5) persons aged 25 - 64 years who have medical conditions that put them at higher risk for influenza complications. In the United States, these five groups contain an estimated 159 million people.

If the initial supply fails to meet population demand in specific geographic areas, the Advisory Committee has developed a plan to target a subset of the five initial target groups. These subset groups are comprised of: (1) pregnant women, (2) persons who live with or provide care for infants $<6$ months, (3) health care and 
emergency medical services personnel, (4) children aged 6 months -4 years, (5) children and adolescents aged 5-18 years who have medical conditions that put them at higher risk for influenza related complications. It is estimated that these subset groups would comprise approximately 42 million people in the United States. As the initial supply increases, decisions to expand vaccination outside of the target groups should be made based on the judgment of state and local health officials to best meet the needs of a specific region.

\section{Infant Considerations}

Infants are at high risk for influenzarelated illness. It is well known that breastfed infants are less susceptible to respiratory illness when compared to bottle fed infants. Mothers should be highly encouraged to breastfeed their infants. The CDC additionally recommends that unnecessary formula supplementation be eliminated so that the infant receives as much maternal antibody as is possible. Ideally, only healthy adults should care for infants. This includes infant feeding. The risk for transmission of influenza H1N1 through breast milk remains unknown. However, reports of seasonal flu viremia have shown that the risk of virus crossing into breast milk is rare. Theoretically, this should also be true for influenza H1N1. Both oseltamivir and zanamivir are considered to be compatible with breastfeeding. Women infected with H1N1 should be encouraged to express their milk so that healthy adults can bottle feed the infants with antibody rich breast milk.

\section{Summary of Current Recommendations}

1. Pregnant women are at high risk for influenza related complications

2. Testing for influenza is not always necessary for a pregnant woman with fever and respiratory symptoms suspicious for flu; especially if she has had contact with others with confirmed H1N1. Treatment decisions should not be based on a negative rapid test. Testing should be considered in hospitalized patients or if the diagnosis is uncertain. Rapid influenza testing is available but is poorly sensitive. If necessary, the PCR testing for H1N1 is available thru the University of lowa Hygienic Lab (please see website below).

3. Begin treatment with empiric antiviral therapy as soon as possible and preferably within the first 48 hours of symptom onset. For women who have had symptoms beyond two days there is evidence that antiviral therapy may still be beneficial.

4. Oseltamavir is considered the first line agent for the treatment of H1N1 influenza. This drug is administered orally and has improved systemic absorption when compared to zanamivir. Side effects are fairly minimal with the most common reported being nausea and vomiting.

5. Zanamivir is also acceptable for the treatment of $2009 \mathrm{H} 1 \mathrm{~N} 1$. The route of administration is inhalation and has decreased systemic absorption when compared to oseltamivir. Side effects include bronchospasm and may lead to 
respiratory complication in persons with underlying respiratory disease

6. Pregnant women who have been in close contact with individuals who have symptoms of influenza-like illness should receive post-exposure chemoprophylaxis.

7. For chemoprophylaxis in pregnant women, a preferred antiviral has not been determined. Zanamivir is generally not recommended in persons with underlying lung disease such as asthma and chronic obstructive pulmonary disease.

8. 2009 H1N1 Vaccine should be available by mid to late October. All pregnant women should be highly encouraged to get the H1N1 vaccine as well as the seasonal vaccine.

9. Preliminary data suggests that older children and adults will only require one dose of H1N1 vaccine to acquire immunity

10. Simultaneous administration of inactivated vaccines against seasonal and $2009 \mathrm{H} 1 \mathrm{~N} 1$ is acceptable if different anatomic sites are used.

11. Using data from seasonal influenza viremia, the risk of $\mathrm{H} 1 \mathrm{~N} 1$ virus crossing into breast milk is considered rare. The CDC highly encourages breastfeeding from both healthy and infected mothers so that the infant receives as much maternal antibody as is possible.

12. Only healthy adults should be caring for infants. It is recommended that infected mothers should express their breastmilk and allow a healthy adult to bottle feed the infant the expressed breastmilk.

13. Prevention is the key to controlling the 2009 H1N1 influenza pandemic. The Centers for Disease and Prevention Guidelines are as follows:

a. Wash hands frequently with soap and water or use alcohol-based hand cleaner

b. Cover your mouth and nose with a tissue when coughing or sneezing

c. Avoid touching your eyes, nose and mouth

d. People who are sick with an influenza-like illness should stay home and avoid travel for at least 24 hours after fever is gone.

e. Avoid close contact (within about 6 feet) with persons with influenza like symptoms

f. The effectiveness of respirators and facemasks in preventing transmission of influenza virus is not known. Use of a facemask or respirator is of most benefit if used consistently when exposed to an ill person.

g. Respirators (such as N95 or high filtering device) require fitting, medical clearance. Facemasks may be considered as an alternative to respirators.

h. Use of respirators of facemasks generally are not recommended for workers in non-healthcare settings for general work activities 
i. Ill persons should be placed in well ventilated areas when possible and placed at least 6 feet away from other people.

14. KEY - Information regarding 2009 Influenza $\mathrm{H} 1 \mathrm{~N} 1$ is evolving rapidly. Recommendations are frequently updated as more information is learned regarding $2009 \mathrm{H} 1 \mathrm{~N} 1$. Please refer to the websites below to stay updated regarding the latest information.

\section{Websites}

Federal Sites:

- Agency for Healthcare Research and Quality: Tools and Resources for Influenza Preparedness http://www.ahrq.gov/prep/swineflu.htm

- Centers for Disease Control \& Prevention (CDC): H1N1 Flu (Swine Flu) http://www.cdc.gov/h1n1flu

- National Library of Medicine (NLM) EnviroHealth Links: 2009 H1N1 Flu

http://sis.nlm.nih.gov/enviro/swineflu.html

\section{State Sites:}

- CDC Public Health Resources: State Health Departments (MMWR):

http://www.cdc.gov/mmwr/international/relres.ht

$\mathrm{ml}$

Select state to go to that state's general health department website.

- Iowa Department of Public Health

www.idph.state.ia.us/

Professional Organization:

- American College of Obstetricians and Gynecologists (ACOG)

www.acog.org

\section{References}

1. Swine influenza $A(H 1 N 1)$ infection in two children - Southern California, March-April
2009. MMWR Morb Mortal Wkly Rep 2009;58:400-2.

2. Jamieson DJ, Honein MA, Rasmussen SA, Williams JL, Swerdlow DL, Biggerstaff MS. H1N1 2009 influenza virus infection during pregnancy in the USA. Lancet 2009;374(9688):451-8.

3. Rasmussen SA, Jamieson DJ, Bresee JS. Pandemic influenza and pregnant women. Emerg Infect Dis. 2008 Jan;14(1):95-100.

4. Freeman DW, Barno A. Deaths from Asian influenza associated with pregnancy. Am J Obstet Gynecol 1959;78:1172-75.

5. Pregnant women and novel influenza $A$ (H1N1) virus: considerations for clinicians, June 30, 2009. Centers for Disease Control and Prevention.

6. Use of influenza A (H1N1) 2009 monovalent vaccine - recommendations of the advisory committee on immunization practices (ACIP), 2009. MMWR Morb Mort Wkly Rep;58:1-8. 